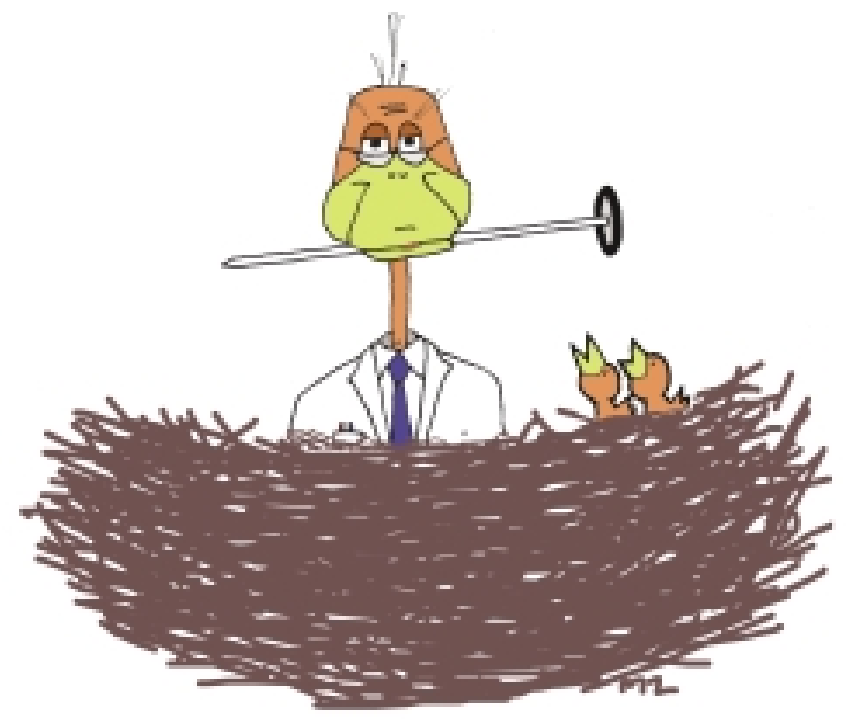

Levodopareducesthesymptomsof Parkinson'sdisease, butmight it hasten neurodegeneration? A randomized placebo controlled trial evaluated 361 patients with early Parkinson's disease who received varying amounts of carbidopa-levodopa or a matching placebo for 40 weeks after which treatment was withdrawn for 2 weeks. Theseverity of parkinsonism increased morein the placebo group than in those receiving levodopa. Interestingly, neuroimaging studies done to assess striatal dopamine transporter density suggested that levodopaperhapsacceleratestheloss of nigrostriatal dopamine nerve terminals. These contrasting results mean that our question remainsunanswered.

New England J ournal of M edicine2004, 351, 2498-508.

Research done on over 100 patients and controls in Rochester, USA and Japan identifies N M O-I gG as a specific serum marker autoantibody of neuromyelitis optica (Devic's disease). This condition needs to be differentiated from multiple sclerosis for several reasons but also because the treatment for the two can vary. Immunosuppressive drugs (azathioprine and corticosteroids) are recommended for neuromyelitis optica, whereas immunomodulatory drugs (interferon beta and glatiramer acetate) are recommended for early treatment of multiple sclerosis. So a serum test to differentiate the two conditionsis welcome.

Lancet 2004, 364, 2106-12.

M etoclopramidehas been used for thetreatment of nauseaassociated with migraine, and case reports have suggested that patients get substantial pain relief even before they have received an analgesic. Now a meta-analysis, which reviewed 13 trials involving 655 adultsfindsthat metoclopramidewasmorelikelyto providesignificantreduction in migrainepain compared to placebo. Theauthors suggest that metoclopramideshould be considered as the primary agent in treating acutemigrainein emergency departments. BMJ 2004, 329, 1369-73.

Researcherswereableto isolatestem cellswith myogenicand neurogenic propertiesfrom samplesfrom thebrachioradialismuscle

\section{CARPHOLOGY BY RAJENDRA}

of 12 patients undergoing coronary artery surgery in whom the radial artery was being used as a conduit. M uscle may therefore bea source of pluripotent stem cells for cell based treatments for myogenic and neurogenic diseases. Fetal tissueis a rich source of stem cells, but its use raises several ethical issues, and hence an ethically acceptable source of stem cells is al ways good news. Lancet 2004, 364, 1872-83.

After a stroke, many people do not get out of the house as much asthey would likebutan occupational therapy programmecould help them do just that.Theprogrammewastested in arandomised controlled trial involving 168 community dwelling people with a stroke. Participants in the programme were more likely to get out of the house as often as they wanted. Some reasons for poor outdoor mobility are remediable, say the authors. These include lack of confidence and inadequate information on transport options, aids, appliances, or adaptations to thehome.

BM J 2004, 329, 1372-5.

A study designed to estimate the incidence of rhabdomyolysis in patients treated with different statins and fibrates, finds that risk of rhabdomyolysis wassimilar and low formonotherapy with atorvastatin, pravastatin, and simvastatin. Combining a statin and a fibrate increased the risk, especially in older people with diabetes mellitus. In all, 24 cases of hospitalised rhabdomyolysis occurred in over 250000 patients treated with lipid lowering agents. Fluvastatin and lovastatin were excluded from the final analyses because too few patients were on them.

Journal of theAmerican M edical Association 2004, 292, 2585-90.

Publication bias means that studies with negative results are likely to be rejected by journal editors. But you need not despair if you have ended up with a negative result for the Journal of Negative Resultsin Biomedicine should welcomeyour paper. For example, it reports that levels of $\mathrm{S100B}$, which belongs to a family of calcium binding proteins, werenot associated with either disability or M RI findings in primary progressive multiple sclerosis and that interferon -1a has no effect on S100B levels. The authors assessed 50 patients recruited in a phaselI trial of IFN -la over 2 years.

http://www.jnrbm.com/content/3/1/4.

A debate piece in the Christmas issue of the BM J argues that the human eye is incapable of applying the offside rule correctly in a football game. To do this, argues the author, the referee must be able to keep in his visual field at least five objects at the same time - two players of theattacking team, the last two players of the defendingteam, and theball. Thisisbeyond thecapacity of the human eye, which says theauthor may explain why many offside decisions are controversial.

BMJ 2004, 329, 1470-2. 